\title{
Efficacy and safety of intravenous bisphosphonates beyond two years
}

\author{
E Gilliam, J Winters, III, E McGuire, S Lee, M Purdy, M Fekrazad, M Royce, K \\ Choi
}

\section{Citation}

E Gilliam, J Winters, III, E McGuire, S Lee, M Purdy, M Fekrazad, M Royce, K Choi. Efficacy and safety of intravenous bisphosphonates beyond two years. The Internet Journal of Oncology. 2009 Volume 7 Number 2.

DOI: $\underline{10.5580 / 2998}$

\begin{abstract}
Background: Metastatic bone disease, a common complication of malignancy, causes significant morbidity in affected patients. Intravenous bisphosphonates (IBPs) have shown efficacy in preventing skeletal morbidity, however few studies have investigated their efficacy and safety in cancer patients beyond 2 years of use. This retrospective study documents long term clinical use of IBPs among a variety of patient populations and clinical settings. Methods: This study is a multi-center retrospective chart review of patients who received IBPs (Pamidronate or Zoledronic Acid) for more than 24 months. Patients were at least 18 years of age and had tumor-associated bone disease. Data analysis focused on skeletal related events (SREs) and drug related toxicities such as renal failure and osteonecrosis of the jaw (ONJ). Results: Ninety-two patients were included with the most common diagnosis being multiple myeloma. A total of 44 SREs occurred among 23 patients. Mean time to first SRE was 515 days. The rate of skeletal events in the first 2 years of treatment was 0.70 , while skeletal morbidity rate (SMR) in subsequent years was 0.16 . Thirteen patients experienced renal toxicity; most patients' renal function normalized after brief or permanent cessation of IBP therapy. ONJ was associated with 5 patients. Conclusion: Continuation of IBP therapy for skeletal metastasis after 2 years of treatment may improve skeletal morbidity. Similar to the first 2 years of treatment, significant toxicities such as renal failure and ONJ are uncommon.
\end{abstract}

\section{INTRODUCTION}

Bone metastasis leads to significant morbidity, such as bone pain, pathologic fractures, spinal cord compression, and hypercalcemia of malignancy. ${ }^{1,2}$ Optimal management of bone metastasis is important as it may significantly improve quality of life, while reducing healthcare-associated cost and resources. This may be especially true for patients receiving novel cancer therapies which have been successful in prolonging survival.

Intravenous bisphosphonates (IBPs) have been proven effective in reducing skeletal related events (SREs) by several randomized trials. Two IBPs [Zoledronic Acid (Zometa ${ }^{\circledR}$, Novartis) and Pamidronate (Aredia ${ }^{\circledR}$, Novartis)] are currently available and have FDA approval for use in metastatic bone disease. These drugs inhibit tumor mediated activation of osteoclasts and thus reduce bone resorption. In general, IBPs are well-tolerated; however, serious adverse reactions such as renal failure and osteonecrosis of the jaw
(ONJ) can occur in a small minority of patients. ${ }^{3-12}$ Large randomized trials have evaluated the renal safety of IBPs in patients with skeletal metastasis and found that renal failure is rare when the IBPs are administered at the recommended doses and infusion rates. ${ }^{13-16}$

No randomized controlled trial of IBPs has evaluated the efficacy or safety of use beyond two years. As better cancer therapies allow patients to live longer with metastatic disease, it becomes more important to know the optimal duration of therapy with these bone-targeting agents. Without supporting evidence to clearly direct duration of therapy, the decision to continue treatments is often at the discretion of the clinician. The American Society of Clinical Oncology (ASCO) recommends continuing IBP therapy in women with breast cancer until a substantial decline in the patient's general performance status based on clinical judgment. ${ }^{17}$ In multiple myeloma patients, ASCO recommends discontinuing bisphosphonate therapy for 
patients with responsive or stable disease, or to continue therapy at the clinician's discretion. ${ }^{18}$ Due to the relative scarcity of evidence regarding the safety and efficacy of continuing IBPs in patients with skeletal metastasis who survive beyond two years, further investigations are needed to evaluate the optimal duration of treatment.

\section{METHODS}

We conducted a multi-center retrospective chart review of cancer patients receiving IBPs for metastatic bone disease for a duration greater than twenty-four months. Institutional review boards approved the study at three sites located in Albuquerque, New Mexico: The University of New Mexico Cancer Center, The Albuquerque Veterans Affairs Medical Center Hematology/Oncology Clinic, and the private practice group Hematology Oncology Associates. Patient eligibility criteria included being of 18 years of age or older, having radiographic and/or cytologic confirmation of bone metastasis, receiving treatment with intravenous

Pamidronate or Zoledronic Acid at regular intervals for more that 24 months, and having sufficient follow-up data throughout the course of treatment.

Demographic information included ethnicity, gender, age, baseline height and weight, baseline performance status, baseline pain level, cancer type and stage at diagnosis, date of diagnosis of bone metastasis and distribution of bone metastasis. Treatment information included, IBP drug used, dose, infusion dates, duration of use, total number of infusions received, reason for discontinuation, any documented SRE's and concomitant anti-cancer therapy.

Efficacy was measured by the incidence of SREs, including pathologic fracture, spinal cord compression, or hypercalcemia of malignancy. If two SREs occurred in the same 21 day period, only the first event was counted to ensure that linked events were not counted as separate occurrences. Efficacy parameters included characterization of SREs occurring in the first two years and beyond two years of IBP treatment, time to first SRE, and skeletal morbidity rate (SMR).

Renal toxicity was determined by changes in serum creatinine. A significant increase of serum creatinine was defined as an increased by $\geq 0.5 \mathrm{mg} / \mathrm{dl}$ with a baseline $\leq$ $1.4 \mathrm{mg} / \mathrm{dl}$, by $\geq 1.0 \mathrm{mg} / \mathrm{dl}$ with a baseline $\geq 1.4 \mathrm{mg}$, or if the baseline doubled. Each patient's serum creatinine was documented at baseline, prior to each administration of IBP, and at discontinuation of therapy or last known evaluation. T-test comparison was used to for statistical comparison of the mean baseline serum creatinine measurement, highest mean creatinine measurement, and final mean creatinine measurements. Diagnosis of ONJ was determined solely by documentation in physician progress notes stating if the patient developed or experienced the condition.

\section{RESULTS}

A total of 92 patients met study inclusion criteria. The majority of our patients were white males $(62 \%)$, and the most common cancer diagnosis was multiple myeloma (43\%). Additional patient and treatment characteristics are presented in Tables 1 and 2, respectively.

\section{Figure 1}

Table 1: Patient Characteristics

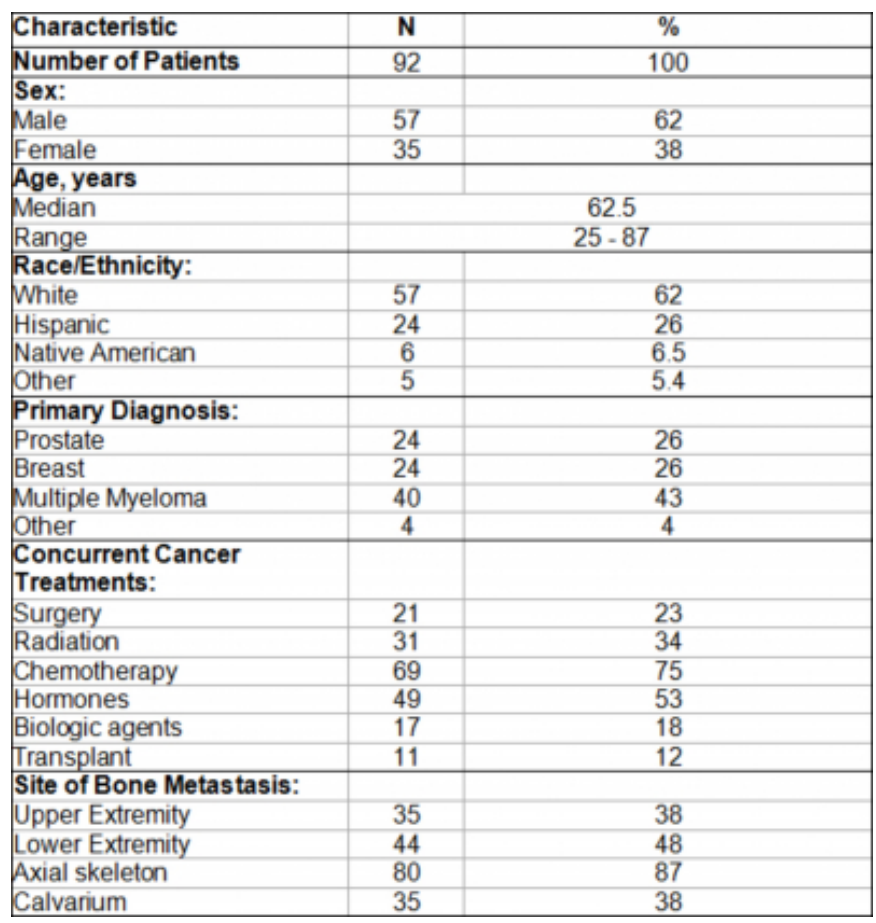

\section{Figure 2}

Table 2: IBP Treatment characteristics

\begin{tabular}{|l|c|c|}
\hline Treatment Characteristics & Median & Range \\
\hline Number of Treatments & 36 & $13-97$ \\
\hline Duration of Treatments, Months & 42.1 & $24.7-128$ \\
\hline Frequency of Treatments, Weeks & 4.8 & $3.5-15$ \\
\hline Reasons for Discontinuation & $\mathbf{N}$ & $\%$ \\
\hline Hospice/Death/Performance Status & 40 & 43.5 \\
\hline Renal Toxicity & 5 & 5.4 \\
\hline Osteonecrosis of the Jaw & 5 & 5.4 \\
\hline Other/ Un-Documented & 17 & 18.5 \\
\hline Patients Continuing Treatments & 25 & 27.2 \\
\hline
\end{tabular}

A total of 44 SREs occurred among 23 (29.3\%) patients. Seventeen $(74 \%)$ patients developed an SRE in the first two years of treatment, while 6 (24\%) experienced an SRE after two years of treatment. Mean time to first SRE was 515 days 
(95\% confidence interval: 248 - 782). Overall SMR in the first two years was 0.70 , while SMR in subsequent years was 0.16 .

The most common reason for discontinuation of IBP therapy was deterioration of patient's condition due to disease progression. Several records failed to identify a reason for discontinuation of treatment. Furthermore, the physician's decisions to discontinue IBP treatments in these patients were not associated temporally with changes in the patient's performance status or development of adverse drug effects. Many patients' IBP treatments had yet to be discontinued at time of chart review.

Significant renal toxicity and ONJ were both uncommon and reported as reason for treatment discontinuation in only 10 patients. Significant differences were found between the mean baseline and mean highest creatinine levels $(\mathrm{p}<0.001)$, but no significant differences were found between the baseline and final creatinine levels $(\mathrm{p}=0.0713)$. Of the 13 patients who experienced renal toxicity (of all grades), serum creatinine returned to normal in 9 of the patients after brief or permanent cessation of IBP therapy. Only five patients permanently discontinued IBP treatments secondary to developing renal toxicity. Additionally, physicians suspected or documented a diagnosis of ONJ among five patients. No other unexpected significant toxicities related to IBP treatment were reported beyond 2 years of use apart from renal toxicity and $\mathrm{ONJ}$.

\section{DISCUSSION}

This retrospective study documents clinical use of IBP despite limited evidence or guidelines endorsing such use in three different practice settings: a military facility, an academic facility, and private practice. Clinicians have chosen to continue IBP treatments to many patients $(43.5 \%)$ until death or significant decline in performance status. Physicians infrequently attributed drug toxicity, either renal dysfunction (5\%) or ONJ (5\%), as reason to discontinue treatment. Although the median duration of therapy is 3.5 years, we documented patients receiving treatment for as long as 10 years. Although the interval between treatments increased over time, the reason for lengthening the time interval between treatments was poorly documented by physicians.

As expected, the SREs occurred less frequently as treatments continued. Although multiple factors may have influenced the incidence of SREs overtime, we have little reason to suspect there was a loss of efficacy of IBP therapy after two years. Until more rigorous studies are conducted that prove otherwise, we conclude from our study that patients benefit from continued IBP treatment.

There does not seem to be a trend towards a cumulative renal toxicity as there was no significant difference between initial and final serum creatinine values. Our patient population had multiple risk factors for developing renal toxicity such as their underlying malignancy, use of cytotoxic chemotherapy, advanced age, and medical comorbidities; yet, almost all elevations in creatinine returned to baseline after brief or permanent cessation of IBP therapy. The relatively low incidence of renal toxicity observed in this study suggests that IBPs have little nephrotoxicity even beyond two years of use.

The mechanism of toxicity and predisposing risk factors for developing ONJ merits further research; however, it is reassuring to note that the rates remain low even among patients with prolonged IBP treatments. Establishing the pathophysiology underlying ONJ and identifying predisposing factors may assist in recognizing which patients are at increased risk. Many questions regarding the risks of ONJ and use of IBP remain unanswered and fall outside of the scope of this study

It is important to note our study is consistent with two previously published retrospective reviews in terms of maintained efficacy and low incidence of toxicity. ${ }^{19,20}$ In contrast to those studies, we evaluated IBP use among a more diverse patient population with a higher percentage of males diagnosed with multiple myeloma and a larger representation of minorities. This is an important distinction considering the possible confounders of gender and underlying diseases on skeletal morbidity. In addition to confirming previously documented results, our study strengthens the ability to generalize such results to a larger patient population.

In short, this study provides additional evidence suggesting patients benefit from IBP therapy beyond two years without an increased risk of developing serious toxicities. While research is needed to determine the true efficacy of prolonged IBP treatments, clinicians are choosing to continue treatments in hopes that benefits outweigh known risks.

\section{References}

1. Coleman RE: Metastatic bone disease: clinical features, pathophysiology and treatment strategies. Cancer Treat Rev 27:165-176, 2001 
2. Jemal A, Tiwari RC, Murray T, et al: Cancer statistics. CA Cancer J Clin 54:8-29, 2004

3. Durie BG, Katz M, Crowley J. Osteonecrosis of the jaw and bisphosphonates. N Engl J Med 353:99-102, 2005

4. Bounameaux HM, Schifferli J, Montani JP, et al: Renal

failure associated with intravenous diphosphonates. Lancet $1: 471,1983$

5. Markowitz GS, Appel GB, Fine PL, et al: Collapsing focal segmental glomerulosclerosis following treatment with high-dose pamidronate. J Am Soc Nephrol. 12:1164-1172, 2001

6. Markowitz GS, Fine PL, Stack JI, et al. Toxic acute tubular necrosis following treatment with zoledronate (Zometa). Kidney Int 64:281-289, 2003

7. Zazgornik J, Grafinger P, Biesenbach G, et al: Acute renal failure and alendronate. Nephrol Dial Transplant

12:2797-2798, 1997

8. Banerjee D, Asif A, Striker L, et al: Short-term, high-dose pamidronate-induced acute tubular necrosis: the postulated mechanisms of bisphosphonate nephrotoxicity. Am J Kidney Dis 41:E18, 2003

9. Barri YM, Munshi NC, Sukumalchantra S, et al: Podocyte injury associated glomerulopathies induced by pamidronate. Kidney Int 65:634-641, 2004

10. Zojer N, Keck AV, Pecherstorfer M: Comparative tolerability of drug therapies for hypercalcaemia of malignancy. Drug Saf 21:389-406, 1999

11. Dumon JC, Magritte A, Body JJ: Efficacy and safety of the bisphosphonate tiludronate for the treatment of tumorassociated hypercalcemia. Bone Miner 15:257-266, 1991

12. Ruggiero SL, Mehrotra B, Rosenberg TJ, et al:

Osteonecrosis of the jaws associated with the use of bisphosphonates: a review of 63 cases. J Oral Maxillofac Surg 62:527-534, 2004

13. Rosen LS, Gordon D, Kaminski M, et al: Long-term efficacy and safety of zoledronic acid compared with pamidronate disodium in the treatment of skeletal complications in patients with advanced multiple myeloma or breast carcinoma: a randomized, double-blind, multicenter, comparative trial. Cancer 98:1735-1744, 2003 14. Rosen LS, Gordon D, Tchekmedyian NS, et al: Longterm efficacy and safety of zoledronic acid in the treatment of skeletal metastases in patients with nonsmall cell lung carcinoma and other solid tumors: a randomized, Phase III, double-blind, placebo-controlled trial. Cancer

100:2613-2621, 2004

15. Saad F, Gleason DM, Murray R, et al: A randomized, placebo-controlled trial of zoledronic acid in patients with hormone-refractory metastatic prostate carcinoma. J Natl Cancer Inst 94:1458-1468, 2002

16. Kohno N, Aogi K, Minami H, et al: Zoledronic acid significantly reduces skeletal complications compared with placebo in Japanese women with bone metastases from breast cancer: a randomized, placebo-controlled trial. J Clin Oncol 23:3314-3321, 2005

17. Hillner BE, Ingle JN, Chlebowski RT, et al: American Society of Clinical Oncology 2003 update on the role of bisphosphonates and bone health issues in women with breast cancer. J Clin Oncol 21:4042-4057, 2003

18. Kyle RA, Yee GC, Somerfield MR, et al: American Society of Clinical Oncology 2007 clinical practice guideline update on the role of bisphosphonates in multiple myeloma. J Clin Oncol 25:2464-2472, 2007

19. Crawford BS, McNulty RM, Kraut EH, et al: Extended use of intravenous bisphosphonate therapy for the prevention of skeletal complications in patients with cancer. Cancer Invest 27:984-988, 2009

20. Guarneri V, Donati S, Nicolini M, et al: Renal safety and efficacy of i.v. bisphosphonates in patients with skeletal metastases. Oncologist 10:842-848, 2005 


\section{Author Information}

Eric H. Gilliam, Pharm.D.

University of New Mexico Hospital

\section{John P. Winters, III, M.D}

University of New Mexico Hospital

Elizabeth A. McGuire, M.D.

Raymond G Murphy VA Medical Center

\section{Sang-Joon Lee, Ph.D}

Division of Epidemiology and Biostatistics, University of New Mexico

\section{Malcolm Purdy, M.D}

Hematology/Oncology Associates

\section{Houman Fekrazad, M.D}

University of New Mexico Cancer Center

Melanie E Royce, M.D., Ph.D.

University of New Mexico Cancer Center

Kook I. Choi, Ph.D

Department of Data Science, Inje University 